\title{
Post-operative Analgesic Use and Analysis of Related
}

\section{Factors}

\author{
Deniz Hayat $^{1}$ (D) , Fadime Çınar ${ }^{2}$ (D), Fatma Eti Aslan 3 (D) \\ 1 Kocaeli University Research and Application Hospital, Intensive Care Unit, Kocaeli, Turkey; denizabnd@hotmail.com \\ (D.H.); \\ 2 Nişantaşı University School of Health Sciences, Department of Nursing, İstanbul, Turkey; fadime.cinar@hotmail.com \\ (F.C.); \\ 3 Bahçeşehir University Faculty of Health Sciences, Department of Nursing, İstanbul, Turkey; fatmaetiaslan@gmail.com \\ (F.E.A.); \\ * Correspondence: denizabnd@hotmail.com (D.H.);
}

Scopus Author ID 57204505449

Received: 3.06.2021; Revised: 15.08.2021; Accepted: 18.08.2021; Published: 4.09.2021

\begin{abstract}
Pain is a problem that humanity has experienced since its existence and is still seeking a solution. Despite all the advances in pharmacology and technology in recent years, surgical pain remains a serious problem. This study was carried out to determine the analgesic use status and related factors according to the patients' pain levels after surgery. The study consisted of 188 patients hospitalized for surgical intervention in a private hospital operating in the province of Istanbul between 01.01.2019 and 31.12.2019 and followed up on analgesic use in the clinic for 3 days after the surgical intervention. Surgical pain averages of the patients who underwent surgery were compared according to the patient's descriptive characteristics regarding the day of the surgery. According to this comparison, it was found that the type of surgical intervention and the character of the pain affects the incidence of pain score on the first day of surgery. It was determined that on the 1st day after surgery, gender, age, alcohol use affected the incidence of pain, and on the 2nd day, there was a significant difference between the pain averages of alcohol substance use and anesthesia type. It was determined that the rate of patient-controlled analgesia use was $42 \%$ on the day of surgery, $14.4 \%$ on the 1 st day, and $7.4 \%$ on the 2 nd day. It was determined that the mean pain score did not differ between patients who received and did not receive patient-controlled analgesia on the 1 st and 2nd days after surgery. As a result, it was determined that the frequency of experiencing surgical pain was high despite the development in pharmacological treatments and evidence-based non-pharmacological methods. Nurses play a very important role in providing painlessness and comfort with the adequate use of analgesic drugs. It is thought that nurses and doctors must plan pain before surgery, taking into account the individual characteristics of the patients, for the adequate use of analgesics after surgery. For these plans, it is recommended to increase awareness by following up-to-date information on pain treatment.
\end{abstract}

Keywords: pain; post-operative pain; pain management; analgesic drugs.

(C) 2021 by the authors. This article is an open-access article distributed under the terms and conditions of the Creative Commons Attribution (CC BY) license (https://creativecommons.org/licenses/by/4.0/).

\section{Introduction}

Despite improvements in care processes at all stages of surgery, many sequels can develop after major surgical interventions. One of them is pain, which is an inevitable experience after surgery. Pain is an experience caused by an unpleasant stimulus, where emotional and perceptual processes coexist. Pain, which is related to the Latin word poena (punishment, torture), is defined as "an uncomfortable feeling due to illness, bodily injury, or 
organic disorder". Pain is a complex, subjective phenomenon beyond being a physical process [1]. Pain can cause damage to the immune, hormonal and hematological systems, respiratory and cardiac systems, cause movement restriction, lead to sleep or weakness, or even cause agitation, psychosis, aggressive behavior, and delirium [2]. Pain after surgery is an acute form of pain caused by neuronal signals caused by surgical trauma and the associated inflammatory process. It includes a set of unpleasant sensory, emotional, and mental controls linked to the autonomic, psychological, and behavioral responses triggered by surgical damage. Pain after acute surgery has side effects that concern autonomic, endocrine, metabolic, and psychological processes [3]. Failure to manage pain after surgery leads to consequences that affect multiple organ systems, such as decreased anti-inflammatory cytokines, changes in acute-phase proteins, and the coagulation system. There are psychological symptoms of untreated pain, the most important of which is chronic pain syndrome. All these changes are known to increase mortality and morbidity $[2,4,5]$. Pain is the trigger of the stressor response, which the body perceives as a threat and switches to self-defense for whatever reason. Measures are not taken against pain perceived as a stressor trigger, and if it lasts for a long time, physiopathological responses to pain develop in the organism. These responses disrupt the patient's sleep and physical activity, negatively impacting their well-being. These negative effects may persist during the rehabilitation period so that recovery may be delayed [6]. Cardiac arrhythmias, ileus, poor wound healing, and respiratory failure may occur. Hypertension, deep vein thrombosis, tachycardia, an increase in oxygen consumption, a decrease in the stomach and intestinal motility, gastrointestinal tract, genitourinary system, urinary retention, leads to catecholamine and cortisol release in the neuroendocrine system, and a rise in blood sugar as a result of this, negative nitrogen balance, the tendency to infection, wound healing delay, spasm and skeletal system, inactivity and psychologically fear, anxiety and insomnia can be seen as [6].

With the increase in the population, the number of surgical procedures has increased. Over time, surgical interventions have become less invasive and have contributed greatly to pain management. Among the common goals of healthcare professionals; reducing the incidence of pain, alleviating the severity of pain, minimizing the side effects of analgesics, accelerating post-surgical recovery and preventing complications, and even preventing chronic pain if possible [7].

Pain treatment is a fundamental human right, and patients are not expected to experience surgical pain after surgery is an acute pain characterized by being predictable, and therefore appropriate treatment strategies can be planned. An effective pain therapy reduces the inflammatory response and catecholamine release, endocrine and metabolic response to surgery prevents or decreases stress and anxiety, Nov facilitated mobilization and prevents spasms [8]. The incidence of Deep Vein Thrombosis and pneumonia decreases [9]. Normal nutrition of the patient is provided by preventing nausea and vomiting due to impaired Gastrointestinal system function. By suppressing immune functions and preventing hypoxia, wound healing is accelerated [10]. The cost of care decreases, morbidity and mortality decrease, and the patient is satisfied with the care he receives [11]. Surgical pain, which is not well controlled, turns into chronic pain and allodynia with peripheral and central sensitivity, causing the patient to suffer pain for a lifetime. For this reason, pain, which is shaped by many mechanisms, has often been a matter of curiosity for researchers [12].

In poorly controlled surgical pain, cardiac arrhythmias, ileus, poor wound healing, and respiratory failure may occur. Hypertension, deep vein thrombosis, increase in tachycardia and oxygen consumption, decrease in gastric and intestinal motility in the gastrointestinal system, 
urinary retention in the genitourinary system, increase in catecholamine and cortisol release in the neuroendocrine system, and as a result, increase in blood sugar, negative nitrogen balance, the tendency to infection, delay in wound healing, muscle spasm in the musculoskeletal system, inactivity and psychologically it can be seen as fear, anxiety, and insomnia [13].

Pharmacological agents used in pain control include nonsteroid anti-inflammatory drugs, opioids, and adjuvants. Nonsteroid anti-inflammatory drugs are the most preferred analgesics. The best reason for this is the lack of serious side effects and its role in relieving pain [14]. If the pain is mild and moderate, nonsteroidal anti-inflammatory drugs are preferred first. Opioids, on the other hand, are used in cases such as surgical pain, fractures, terminal illness, cancer pain. But the literature states that patients continue to suffer moderate to severe pain even when opioids are used.

This research was carried out to determine the use of analgesics and relevant factors according to the patients' pain levels after surgery.

Despite the advances in pharmacological treatments and evidence-based nonpharmacological methods, it has been found that the frequency of experiencing surgical pain is high. It is thought that nurses have a great role in providing comfort with adequate analgesic drugs and that nurses should cause pain. Post-surgical pain is an acute form of pain that is expected to decrease gradually in the patient. It requires pain control and teamwork. The fact that nurses follow the patient more closely and for a long time in teamwork brings nurses to a unique role in observing and solving patient problems [15]. Planning by considering the individual characteristics of the patients before the operation. It is recommended to increase the awareness of nurses by following up-to-date information for planning.

\section{Materials and Methods}

The universe of this descriptive study, which has been of a type from past to present, consisted of 360 patients who were hospitalized for surgical intervention between January 1 and December 31, 2019 in a private hospital operating in the city of Istanbul and stayed for 3 days in the clinic for follow up with analgesic use. The sample of the study, on the other hand, consisted of 190 patients who were calculated via the GPower 3.1 program out of the number of universes. However, patient data of 188 patients were included in the study due to incomplete data recording. The data were collected using "Personal Information, Pain Assessment, and Analgesic Use Form". SPPS 25.0 statistics package program was used to evaluate the data. To carry out the study, ethics approval was obtained on November 09.04.2020, 2002.120.IRB3.058, by applying to the Koç University Social Sciences Ethics Committee. In addition, a study permission form was obtained from the hospital management to carry out the research.

\section{Results and Discussion}

\subsection{Individual characteristics of patients.}

In the evaluation of the data obtained from the patient files $(\mathrm{N}=191)$ included in the study, 60.6 percent $(n=114)$ were female, 50 percent $(n=94)$ was between the ages of $30-49$, and 45.2 percent $(n=n)=85)$ were high school graduates in terms of education level, 68.1 percent $(n=128)$ had a chronic disease, 30.9 percent $(n=58)$ used alcohol-substance, 84.6 percent $(n=159)$ did not have a history of psycho-social support. Zheng et al. [16] included 403 men out of 890 orthopedically used patients, 487 men. The mean age was $48.5 \pm 17$ years. 
In addition, in the evaluation of the urgency of the surgical interventions, 95.2 percent $(n=79)$ were planned surgery, 94.4 percent $(n=170)$ had open surgery as surgical intervention, $81.4 \%$ $(n=153)$ It was determined that the surgical intervention was performed under general anesthesia.

In the study of Karadağ Arlı [17] with the participation of 160 patients hospitalized in general surgery, otolaryngology, urology, and orthopedics clinics; 67.5 percent of the patients participating in the study were male, 61.3 percent were married, 29.4 percent were high school graduates, $63.8 \%$ had no previous surgery, 80.6 percent did not have a chronic disease, 35 percent were in the otorhinolaryngology clinic. It was determined that 18.1 percent of them were hospitalized with the diagnosis of nasal septal deviation and underwent surgical intervention, and the mean age of the patients participating in the study was $40.58 \pm 20.4$.

\subsection{Comparison of pain scores according to the post-surgical days and variables.}

According to the patients' data evaluated in Table 1. the mean scores of experiencing pain were determined. According to the results obtained, the mean pain score of the patients on the day of surgery was $6.47 \pm 1.471(\min 2, \max 10)$, the mean pain score on the 1 st day after surgery was $6.26 \pm 1.590(\min 4, \max 10)$, and the mean score on the 2 nd day after surgery. It was determined that the mean daily pain score was $6.40 \pm 1.786(\min 4$, $\max 10)$. When the results were examined, it was determined that the mean pain was the 1st day after the surgical intervention as the lowest day. One of our research questions is "What is the frequency of postsurgery pain"? When the data related to the surgery were evaluated, it was found that the frequency of pain after surgery was 99.4 percent $(n=187)$ on the day of surgery, 32.4 percent $(n=61)$ on the 1 st day, and 19.7 percent $(n=37)$ on the 2 nd day.

Table 1. Mean pain scores of patients undergoing surgical intervention according to the days of surgical intervention.

\begin{tabular}{l|c|c|c|c|c} 
& $\mathbf{N}$ & Minimum & Maximum & Average & Standard Deviation \\
\hline Surgery Day & 187 & 2 & 10 & 6,47 & 1,421 \\
\hline $\begin{array}{l}\text { 1st Day After Surgical } \\
\text { Intervention }\end{array}$ & 61 & 4 & 10 & 6,26 & 1,590 \\
\hline $\begin{array}{l}\text { 2nd Day After Surgical } \\
\text { Intervention }\end{array}$ & 37 & 4 & 10 & 6,40 & 1,786 \\
\hline
\end{tabular}

Lindberg et al. [18], which included 434 colorectal surgery patients, the pain level was found to be 64 percent on the first day of the surgical intervention, 59 percent on the second day, and 51 percent on the third day. In the study conducted by İzveren and Dal [19] with 142 patients who had undergone abdominal surgery, it was stated that $30-70 \%$ of the patients complained of moderate to severe pain. In this study, it was determined that pain was the most common problem on the 1st day of surgery in 73.1 percent of the patients, on the 2 nd day after surgery in 65.7 percent, and on the 3rd day after surgery in 64.2 percent of the patients. In our study, although pain scores on the day of surgery and the first and second days were not statistically significantly different, the mean pain score on the first day of the surgical intervention was found to be higher than on the 1 st and 2 nd days ( $p>0.05)$. The pain frequency and post-surgery pain score averages according to the day of surgery are consistent with the literature. According to these results, it is thought that the incidence of post-surgical pain is an unresolved problem for years. In the study of Arefayne et al. (2020), consisting of patients with mixed emergency trauma surgery; The overall incidence of moderate to severe post-operative pain in the first 24 hours after emergency orthopedic surgery was 70.5 percent [20]. 
When the comparison of the introductory characteristics of the patients and their pain averages were examined on the first day of the surgical intervention; There was a statistically significant difference between group means in surgical pain averages according to the type of surgical intervention $(\mathrm{F}=2.563 ; \mathrm{p}=0.05<0.05)$ and the character of the pain $(\mathrm{F}=2.869$; $\mathrm{p}=0.037<0.03$ ). It was determined that the source of the differences was those who had open surgery and those whose pain was like an electric shock.

Comparison of the descriptive characteristics of the patients with the mean of surgical pain was examined on the 1st day after the surgery; As a result of the analysis, gender $(\mathrm{F}=3.102$; $\mathrm{p}=0.01<0.05)$, age $(\mathrm{F}=2.689 ; \mathrm{p}=0.00<0.05)$, type of anesthesia $(\mathrm{F}=3.113 ; \mathrm{p}=0.01<0.05)$ and alcohol -substance use $(\mathrm{F}=3.563 ; \mathrm{p}=0.01<0.03)$, the difference between the surgical pain averages and the group averages was statistically significant. It was determined that the differences were caused by the female gender, those aged 29 years and younger, those without alcohol-substance use, and the type of general anesthesia.

Comparison of the introductory characteristics of the patients with the mean surgical pain, when the 2nd day after the surgery was examined; As a result of the analysis, the difference between the group averages of anesthesia type $(\mathrm{F}=2.467 ; \mathrm{p}=0.02<0.03)$ and alcoholsubstance use $(\mathrm{F}=3.069 ; \mathrm{p}=0.01<0.03)$ group averages was found to be statistically significant. The differences were determined to be due to the general anesthesia type and those who did not use alcohol-substance use.

Fen Su et al. [21] found that patients aged 50-65 had a longer period of experiencing pain reported that the elderly are more sensitive to pain Robleda et al. reported that younger patients experienced more pain in the early period of surgery [22]. Zheng et al. In the study they conducted with 890 patients who underwent orthopedic surgery in 2017, it was found that the mean pain scores of the patients in the 50-year-old group were higher than the patients in the other age groups [23]. Although the mean pain scores obtained as a result of this study did not differ significantly according to age, it was determined that the mean pain scores increased as the mean age increased. These results are in agreement with the literature.

It has been reported in the literature that patients with a history of frequent alcohol consumption need more opioids for pain control after surgery and opioid-related side effects such as nausea and vomiting are less than those without such a history. It can be caused by different pathologies [24]. Chin Kao et al., in a retrospective study of 4143 patients who had undergone abdominal surgery, frequent alcohol consumption and active smoking were associated with increased opioid consumption for pain control after surgery. Substance addicts tend not to reveal themselves most of the time, so there are few studies in the literature [25]. Alcohol and substance use were included in our study; It was determined that those who used alcohol or drugs used less recurrent analgesics than those who did not. This result, which is different from the studies, is thought to be an obstacle in the expression of substance use by the patients [26].

There are many sources in the literature confirming the relationship between anesthetics used during anesthesia and analgesia and pain. The technical technique for laparoscopic collectorectomy, laparoscopyc append, and scopic total extraperitoneal inguinal hernia operations, consisting of 171 systems, has been demonstrated appropriately and safely. They found that spinal anesthesia is a good alternative to general anesthesia if side effects are low. It is possible to have animals, a high patient case, a long case of surgical intervention, and assistance such as measures for intubation [27]. Gonen et al., 21 renal patients, are obtained from 62 drawings obtained from tubeless percutaneous nephrolithotomy with a tubeless, 
tubeless anesthetic administered percutaneous nephrolithotomy under general anesthesia. The results were found to be significantly higher in general [28]. This application determined that the patients with general anesthesia had more analgesic application with spinal test repetitive. These results are similar to the studies in the literature.

Table 2. Comparison of the pain scores of the patients undergoing surgical intervention according to the postsurgical days and the type of analgesic used $(n=188)$.

\begin{tabular}{|c|c|c|c|c|c|c|c|c|c|}
\hline \multirow[b]{2}{*}{$\begin{array}{l}\text { Analgesic } \\
\text { Type }\end{array}$} & \multirow[b]{2}{*}{$\mathbf{N}$} & \multicolumn{2}{|c|}{$\begin{array}{l}\text { Surgical } \\
\text { Intervention Day }\end{array}$} & \multicolumn{3}{|c|}{$\begin{array}{l}\text { 1st Day After } \\
\text { Intervention }\end{array}$} & \multicolumn{3}{|c|}{$\begin{array}{l}\text { 2nd Day After Surgical } \\
\text { Intervention }\end{array}$} \\
\hline & & $\bar{X}$ & SD & $\mathbf{N}$ & $\bar{X}$ & SD & $\mathbf{N}$ & $\bar{X}$ & SD \\
\hline Opioid & 56 & 6.46 & 125 & 7 & 6.52 & 1.66 & 10 & 6.60 & 183 \\
\hline NSAIII & 30 & 7,27 & 1,46 & 11 & 6,72 & 1,27 & 5 & 6,80 & 1,30 \\
\hline Parasetomol & 88 & 6,19 & 1,54 & 26 & 6,00 & 1,72 & 19 & 6,15 & 1,77 \\
\hline $\begin{array}{l}\text { Opioid+NSAI } \\
\dot{\mathrm{I}}\end{array}$ & 9 & 6,67 & 1,41 & 4 & 5,25 & 957 & 2 & 8,00 & 2,82 \\
\hline $\begin{array}{l}\text { Opioid+Paras } \\
\text { etemol }\end{array}$ & 5 & 6,25 & 1,25 & 3 & 6,66 & 1,52 & 1 & 4,00 & 1,83 \\
\hline Total & \multicolumn{3}{|l|}{188} & \multicolumn{3}{|l|}{57} & \multicolumn{3}{|l|}{37} \\
\hline $\mathbf{F}$ & \multicolumn{3}{|l|}{3,184} & \multicolumn{3}{|l|}{0,924} & \multicolumn{3}{|c|}{1,037} \\
\hline $\mathbf{P}$ & \multicolumn{3}{|l|}{$015^{*}$} & \multicolumn{3}{|l|}{424} & \multicolumn{3}{|c|}{, 403} \\
\hline PostHoc & \multicolumn{3}{|c|}{$2>3,(p<0.05)$} & & & & & & \\
\hline
\end{tabular}

When the comparison of the pain scores of the patients who underwent surgical intervention by post-operative days and the type of analgesic used was analyzed (Table 2.), the frequency of analgesic use was 100 percent $(n=188)$ on the day of surgery and 30.31 percent $(n=)$ on the 1st day after surgery. 57), 19.68 percent $(n=37)$ on the 2 nd day of the surgical intervention. In addition, paracetamol was found to be the most 46.80 percent $(n=88)$ as the analgesic type used. When the analysis results were examined, it was determined that there was no significant difference between the averages of the analgesic types used on the 1 st and 2 nd days after the surgical intervention ( $p>0.05)$.

3.3. Comparison of patient-controlled analgesia (PCA) mean scores according to the days following the surgical intervention.

When the comparison of patient-controlled analgesia use according to the characteristics of the pain (first day of the surgical intervention); As a result of the analysis, the difference between patient-controlled analgesia and surgical pain averages $(\mathrm{F}=2.358$; $\mathrm{p}=0.02<0.01)$ and anesthesia type $(\mathrm{F}=3.119 ; \mathrm{p}=0.00<0.01)$ was found to be statistically significant. The differences were determined to be due to the mean surgical pain scores $(6$ points and above) and the type of general anesthesia.

When the comparison of patient-controlled analgesia use according to the characteristics of pain (post-surgical intervention 1st day), One-way analysis of variance (Anova) was performed to determine whether there was a significant difference, as a result of the analysis, the difference between the mean scores of patient-controlled analgesia and surgical pain characteristics was not statistically significant $(\mathrm{p}>0.05)$.

When the comparison of patient-controlled analgesia use according to the characteristics of the pain (2nd day after the surgical intervention); a One-way analysis of variance (Anova) was performed to determine whether there was a significant difference, as a result of the analysis, the difference between the mean scores of patient-controlled analgesia and the characteristics of surgical pain was not statistically significant ( $p>0.05)$. 
In the "Post-operative Pain Management Guide" published by the Turkish Society of Anesthesiology and Reanimation (TARD), it is reported that PCA is superior to intramuscular injection applications, provides effective analgesia, and causes fewer side effects. In addition, the epidural PCA method has been stated as a safe, safe, and non-excessive sensory blocking method, and respiratory depression is rare [29]. Shariati et al. found that the use of PCA after abdominal surgery effectively reduced pain intensity, increased patient satisfaction, and reduced the number of analgesics used [30]. Vengadesh et al., it was determined that epidural PCA and İHKA protocols applied for analgesia in the post-surgical period are safe and effective methods compared to other pain relief methods [31]. Tuncel Tekgül and Babacan conducted a study with 60 patients who had elective lumbar disc herniation operation.

In the study, both analgesic methods were found to be equally effective between patients using opioids and patients using intravenous PCA [32]. In this study, the use of PCA in various surgical interventions was included, and when the days after the surgery were examined, the extent of pain, the character of the pain, the severity, and the score levels of pain were found to be similar in patients with and without patient-controlled analgesia use. Contrary to the literature, the reason the pain levels were found to be similar between patients using and not using PCA in our study is thought to be the diversity of surgical intervention types.

\subsection{Comparison of repeat analgesic use by days after surgical intervention.}

When the comparison of re-application of analgesics according to patient descriptive characteristics (first day of surgical intervention) was examined; As a result of the analysis, the difference between the mean score of patient-controlled analgesia $(F=4.165 ; p=0.074<0.10)$ and the type of surgical intervention and the mean score of alcohol-substance use $(\mathrm{F}=3.302$; $\mathrm{p}=0.069<0.10$ ) was found to be statistically significant. It was determined that the differences were due to the openness of the surgical intervention and those who did not use alcohol or drugs.

When the comparison of re-application of analgesics according to the patient's descriptive characteristics (post-surgical intervention 1st day) was examined; As a result of the analysis, the difference between the mean scores of repeated analgesia use and type of surgical intervention was found to be statistically significant $(\mathrm{F}=3.621 ; \mathrm{p}=0.010<0.05)$. Complementary posthoc analysis was performed to determine the sources of the differences, and it was determined that the difference was due to open surgery. It was determined that patients with open surgical intervention had more analgesic re-administration compared to laparoscopic patients.

When the comparison of re-application of analgesics according to the patient's descriptive characteristics (2nd day after the surgical intervention) was examined; As a result of the analysis, the difference between the mean scores of repeated analgesia use $(\mathrm{F}=3.901$; $\mathrm{p}=0.053<0.10$ ) and type of anesthesia was found to be statistically significant. It was determined that the differences were due to the type of general anesthesia. It was determined that patients who underwent general anesthesia for surgery had more repetitive analgesic administration than patients with spinal anesthesia. Repetitively applied analgesic in the literature is untested, unstained. 


\section{Conclusions}

It is known that there are an estimated 230 million major surgeries per year worldwide. A high level of surgical pain; is important because it is directly related to delirium, chronic pain syndrome, increased hospital stay and increased opioid use. Although the results we obtained partially overlap with the results of previous studies, it is thought that it is important that substance addiction and psychological factors are also included in the characteristics of the sample group, unlike these studies. Do individual effects in the study affect the individual's perception of pain and, subsequently, the level of analgesic use? The question has been asked. The following results were obtained; Age and gender factors were found to affect the perception of pain. The amount of analgesic use increased with increasing age. There was a higher incidence of pain in females than males, and the amount of analgesic use increased accordingly. Individuals with pre-planned surgical intervention had a lower incidence of pain than those who received the emergency intervention. Similarly, the prevalence of pain was found to be higher in surgeries performed with general anesthesia and with the open intervention compared to spinal and laparoscopic surgeries. Based on these results reached by the research; Pre-planning the pain treatment and pain level based on the personal data of the patients, eliminating the deficiencies in pain treatment and care by considering the variables such as cultural characteristics, ethnicity, belief of each patient, including the "Quality Use of Analgesics" during the training periods of the health professionals, surgical intervention It is recommended to follow-up pain and keep records at the discharge of patients who have.

\section{Funding}

This research received no external funding.

\section{Acknowledgments}

This research has no acknowledgment.

\section{Conflicts of Interest}

The authors declare no conflict of interest.

\section{References}

1. Goldberg, D.S.; McGee, S.J. Pain as a global public health priority. BMC Public Health 2011, 11, 2-5, https://doi.org/10.1186/1471-2458-11-770.

2. Marret, E.; Rolin, M.; Beaussier, M.; Bonnet, F. Meta-analysis of intravenous lidocaine and post-operative recovery after abdominal surgery. British Journal of Surgery 2008, 95, 13311338,https://doi.org/10.1002/bjs.6375.

3. Aslan, F.E.; Şahin, S.K.; Secginli, S.; Bülbüloğlu, S. Patient satisfaction with nursing practices about postoperative pain management: A systematic review. Agri 2018, 30, 105-115, https://doi.org/10.5505/agri.2018.96720.

4. Levy, B.F.; Scott, M.J.; Fawcett, W.; Fry, C.; Rockall, T.A. Randomized clinical trial of epidural, spinal or patient-controlled analgesia for patients undergoing laparoscopic colorectal surgery. British Journal of Surgery 2011, 98, 1068-1078,https://doi.org/10.1002/bjs.7545.

5. Şenyüz, Y.K., Koçaşl1, S. Multimodal Analgesia and Nursing Approach in Post-Surgical Pain.Journal of Health Academics 2017, 4, 90-95.

6. Malek, M.; Yousefi, R.; Safari, S.; Seyyedi, S.H.S.; Mottaghi, A. Dietary Intakes and Biochemical Parameters of Morbidly Obese Patients Prior to Bariatric Surgery. Obesity Surgery 2019, 29, 1816-1822, https://doi.org/10.1007/s11695-019-03759-x.

7. Yağc1, Ü.; Saygın, M. Pain Physiopathology.Journal of Süleyman Demirel University Faculty of Medicine2 019, 26, 209-220,https://doi.org/10.17343/sdutfd.444237. 
8. Meissner, W.; Zaslansky, R. A survey of post-operative pain treatments and unmet needs. Best Practice \& Research Clinical Anaesthesiology 2019, 33, 269-286, https://doi.org/10.1016/j.bpa.2019.10.003.

9. Sommer, M.; de Rijke, J.M.; van Kleef, M.; Kessels, A.G.H.; Peters, M.L.; Geurts, J.W.J.M.; Gramke, H.F.; Marcus, M.A.E. The prevalence of post-operative pain in a sample of 1490 surgical inpatients. Eur J Anaesthesiol 2008, 25, https://doi.org/10.1017/S0265021507003031.

10. Koneti, K.K.; Jones, M. Management of acute pain. Surgery - Oxford International Edition 2013, 31, 77-83, https://doi.org/10.1016/j.mpsur.2012.12.004.

11. Kavakci, Ö.; Altuntas, E.; Müderris, S.; Kugu, N. Effects of the preoperative anxiety and depression on the post-operative pain in ear, nose and throat surgery. Indian Journal of Otology 2012, 18, 82-87, https://doi.org/10.4103/0971-7749.100721.

12. Al-Hariri, M.T.; Abualait, T.S. Effects of Green Brazilian Propolis Alcohol Extract on Nociceptive Pain Models in Rats. Plants 2020, 9, 3-9, https://doi.org/10.3390/plants9091102.

13. Safari, S.; Rokhtabnak, F.; Djalali Motlagh, S.; Ghanbari Garkani, M.; Pournajafian, A. Effect of intraperitoneal bupivacaine on post-operative pain in laparoscopic bariatric surgeries. Surgery for Obesity and Related Diseases 2020, 16, 299-305, https://doi.org/10.1016/j.soard.2019.10.028.

14. Çavdar, İ.;:Eti Aslan, F. "Surgical Pain” Pain Nature and Control. Ankara: Academician Bookstore 2014, 185-212.

15. Tat Çatal, A.; Cebeci, F. Cycle of Pain, Anxiety, Depression and Nurse's Role in Lumbar Disc Herniation.Hacettepe University Journal of Nursing Faculty 2020, 7, 74-77.

16. Zheng, H.; Schnabel, A.; Yahiaoui-Doktor, M.; Meissner, W.; Van Aken, H.; Zahn, P.; Pogatzki-Zahn, E. Age and preoperative pain are major confounders for sex differences in post-operative pain outcome: A prospective database analysis. PLOS ONE 2017, 12, 1-14, https://doi.org/10.1371/journal.pone.0178659.

17. Karadağ Arlı, S. Nursing Interventions Regarding Pain Management in Surgical Care.Manisa Celal Bayar Health Sciences Institute Journal 2017, 4, 1013-1021.

18. Lindberg, M.; Franklin, O.; Svensson, J.; Franklin, K.A. Post-operative pain after colorectal surgery. International Journal of Colorectal Disease 2020, 35, 1265-1272, https://doi.org/10.1007/s00384-02003580-4.

19. İzveren, A.; Dal, Ü. Early Period Problems in Patients Undergoing Abdominal Surgery and Nursing Practices for These Problems.Hacettepe University Faculty of NursingJournal 2019, 18, 36-46.

20. Arefayne, N.R.; Seid Tegegne, S.; Gebregzi, A.H.; Mustofa, S.Y. Incidence and associated factors of postoperative pain after emergency Orthopedic surgery: A multi-centered prospective observational cohort study. International Journal of Surgery Open 2020, 27, 103-113, https://doi.org/10.1016/j.ijso.2020.10.003.

21. Su, S.-F.; Liao, Y.-C.; Wu, M.-S. Age and pain as predictors of discomfort in patients undergoing transfemoral percutaneous coronary interventions. Heart \& Lung: The Journal of Cardiopulmonary and Acute Care 2018, 47, 576-583, https://doi.org/10.1016/j.hrtlng.2018.07.001

22. Robleda, G.; Sillero-Sillero, A.; Puig, T.; Gich, I.; Baños, J.E. Influence of preoperative emotional state on post-operative pain following orthopedic and trauma surgery. Revista latino-americana de enfermagem 2014, 22, 785-791, https://doi.org/10.1590/0104-1169.0118.2481.

23. Zheng, H.; Schnabel, A.; Yahiaoui-Doktor, M.; Meissner, W.; Van Aken, H.; Zahn, P.; Pogatzki-Zahn, E. Age and preoperative pain are major confounders for sex differences in post-operative pain outcome: A prospective database analysis. PLOS ONE 2017, 12, 1-14, https://doi.org/10.1371/journal.pone.0178659.

24. Morino, R.; Ozaki, M.; Nagata, O.; Yokota, M. Incidence of and risk factors for post-operative nausea and vomiting at a Japanese Cancer Center: first large-scale study in Japan. Journal of anesthesia 2013, 27, 1824,https://doi.org/10.1007/s00540-012-1468.

25. Karacalar, S.; Turgut, N.; Tekin, E. Anesthesia Applications in Substance Addicts and Problems Encountered in Intensive Care.Okmeydanı Medical Journal 2014 30, 134-142.

26. Kao, S.-C.; Tsai, H.-I.; Cheng, C.-W.; Lin, T.-W.; Chen, C.-C.; Lin, C.-S. The association between frequent alcohol drinking and opioid consumption after abdominal surgery: A retrospective analysis. PLOS ONE 2017, 12, https://doi.org/10.1371/journal.pone.0171275.

27. Uzman, S.; Donmez, T. Laparoscopic Abdominal Surgery Under Regional Anesthesia: A Retrospective Evaluation. Haseki Tıp Bülteni 2017, 55, 205-211, https://doi.org/10.4274/haseki.58076.

28. Gönen, M.; Sarı, R.; Çiçek, T.; Dursun, M.; Öztürk, M.Tubeless Percutaneous Nephrolithotomy Under Spinal And General Anesthesia.Turkish Journal of Urology 2010, 36, 33-37.

29. Turkish Society of Anesthesiology and Reanimation. Anesthesia Practice Guidelines. Post-operative Pain Management. 2006; pp. 2-18.

30. Shariati, A. The Effect of Patient-Controlled Analgesia on Intensity of Post-operative Pain Abdominal Surgeries.Jentashapir Journal of Health Research 2014, 5, 1-6.

31. Vengadesh, G.; Sistla, S.; Smile, R. Postoperative Pain Relief Following Abdominal Operations: A Prospective Randomised Study Of Comparasion of Patient Controlled Analgesia with Conventional Parenteral Opioids.Indian Journal of Surgery 2005, 67,30-35. 
32. Tuncel Tekgün, Z.; Babacan, A. Comparison of the Efficacy of Lornoxicam, Morphine and Meperidine with Patient Controlled Analgesia Method After Lumbar Disc Herniation Operation. Nobel Medicus 2013, 9, 2126. 